\title{
On the Use of Economical Theories and Metrics to Design an Intelligent Active Queue Management in the Internet
}

\author{
Shahram Jamali', Bita Alipasandi², Vahid Rafe ${ }^{3 *}$ \\ 'Computer Engineering Department, University of Mohaghegh Ardabili \\ 2Department of Computer Engineering, Zanjan Branch, Islamic Azad University, Zanjan, Iran \\ ${ }^{3}$ Department of Computer Engineering, Malayer Branch, Islamic Azad University, Malayer, Iran; rafe@iust.ac.ir
}

\begin{abstract}
Active Queue Management (AQM) plays an important role in the Internet congestion control. Random Early Detection (RED) is the most popular active queue management algorithm that is used in the in Internet routers. The effectiveness of RED algorithm highly depends on appropriate setting of its parameters. Moreover, RED uses only the average queue length as a congestion meter to trigger packet dropping as a congestion feedback. Since the average queue length considers only long-term behavior of any queue, this approach fails to see instantaneous changes of the queue and hence its reaction is not fast enough. This paper inspires from the economic principles to design a new congestion control algorithm. This algorithm, called ECORED, enhances the network performance by dynamically tuning of RED's parameters. ECORED introduces two meters i.e. queue length growth velocity and drop rate velocity to measure the congestion level and produce appropriate congestion feedbacks. Based on extensive simulations conducted using Network Simulator-2 (ns-2), we show that ECORED algorithm reduces the packet loss rate and achieves high utilization in compare with RED, ARED and NLRED.
\end{abstract}

Keywords: Active Queue Management, Average Queue Size, Congestion Control, Dropped Packets, Random Early Detection, Utilization

\section{Introduction}

With the rapid growth of the Internet-based services, congestion control has emerged as a major issue in computer and communication network design. Congestion of packets in routers results in poor performance and low reliability of the network. Congestion control provides control and avoids congestion for traffic transmission in the Internet. Congestion control area regards active queue management as an important mechanism to notify traffic sources about the early stages of congestion and thereby avoid the need for strong source reaction due to heavy overload. There are many reasons to worry that the current $\mathrm{AQM}$ schemes in the Internet may be reaching its limits. The design of the $\mathrm{AQM}$ algorithm has received specific attention of researchers, because it is currently the most important bottleneck in the performance of the best-effort Internet. AQM algorithm controls the rate of packets arrival into the queue, by ECN marking or packet dropping to get the congestion signal that controls the source rate ${ }^{1}$. Reducing the number of dropped packets and increasing the utilization of the bottleneck link are the most important goals in view point of the performance of the congestion control algorithms. Also, the performance of AQM algorithms is defined by low queuing delay and suitable fairness.

The RED algorithm ${ }^{2}$ is the most popular AQM mechanism which is recommended scheme of AQM by the Internet Engineering Task Force (IETF) ${ }^{1}$. The performance of RED is very sensitive to the average queue size and initial parameter settings ${ }^{3-6}$, which makes its behavior unpredictable. These lead to the lower utilization and higher packet lost rate.

*Author for correspondence 
Recently active queue management techniques have been proposed to alleviate some congestion control problems for IP networks as well as provide some notion of quality of service. There are several proposals on AQM by configuring the RED's drop probability function. For example, $\mathrm{LRED}^{7}$ takes into account the dropping probability based on loss rate and queue length rather than average queue size. This algorithm outperforms stability of original RED and archives high throughput. But, this method introduces additional parameters that are needed to be optimized to stabilize the queue length in different network scenarios. Some other algorithms did not change the basic idea of RED, and tunes its control parameters ${ }^{4,58-12}$. One example is Adaptive-RED ${ }^{5}$ that by using an exponentially weighted moving average as an integral controller, attempts to stabilize router queue length at a level independent of the active connections. Proportional Derivative RED controller (PD-RED) ${ }^{13}$ that proposed by Sun et al. is a new RED scheme based on the proportional derivative control theory that improved the performance of the AQM. However, neither Adaptive-RED nor PD-RED provides any systematic technique to configure the RED parameters. In addition, in both methods the control gain selection is only based on simulation analysis and empirical observation, which can only work well in certain given scenario. Recently, some enhanced algorithms use the queue length and input rate jointly to attain better performance. In ${ }^{8}$, the authors proposed PI that with attention to the queue mismatch regulates the queue length to an expected value. If the network states are known a priori, PI optimal parameters will be determined through a control theoretical model. However, in dynamic networks, PI could have to use a conservative setting to ensure stability, that yielding long response time. $\mathrm{REM}^{14}$, in order to calculate the drop probability, uses a linear combination of the queue mismatch and input rate mismatch, that input rate mismatch, is equivalently simplified to the queue variance between two adjacent length samples. Each of these approaches, however, has its own limitations and disadvantages. Therefore, a new controller design with better performance and stability seems necessary.

Barrera et al. proposed a novel likelihood-based congestion detector with improved detection and false alarm probabilities ${ }^{15}$. Although this statistical detector of congestion proved to outperform previously proposed ones and also reduced detection time, the congestion measurements need to be properly mapped into congestion notifications. Poorly placed congestion marks not only impacts the efficiency of the network, but also the statistical distribution of the routers' buffers. Yousefi zadeh et al. present the results of systematic study on optimal fine tuning of RED parameters and as result, they have identified the RED parameters locally minimizing the loss characteristic of the queue while satisfying an acceptable delay profile ${ }^{16}$. Classification and performance evaluation of the different variants of the AQM schemes are presented in ${ }^{17}$.

In recent years, a number of approaches using economic models for resource allocation have been proposed, which include ${ }^{18-23}$. Some of them divide the traffic into multiple priority classes and use a fixed price for each class. A better approach may be to use dynamic pricing where the price depends on network activities such as congestion. Wang and Schulzrinne ${ }^{18}$ proposed a strategy where the price depends on the service class's average demand. This strategy requires resource reservation which obviously would incur some amount of overhead. Another instance is the pricing model of Bagliet to et al. ${ }^{19}$ that the same amount of bandwidth is assigned to all traffic classes. Although simple, this approach may not be that reasonable as different customers may have different demands for bandwidth. The pricing model in the scheme proposed in ${ }^{20}$ is governed by a well-defined statistical model based on the source traffic. However this scheme does not take into account dynamic changes in the traffic. The authors in ${ }^{21}$ offer the Economic Generalized Particle Model (EGPM) approach to intelligent allocation of network bandwidth that it is a considerable extension of the Generalized Particle Model (GPM) ${ }^{22}$. The approach encompasses two major parts: dynamic allocation of network bandwidth based on GPM and dynamic modulation of price and demands of network bandwidth. In ${ }^{23}$, the theory of consumer-firm developed in microeconomics has been considered as a model of the non-strategic behavior that adopted in order to exploit the natural selfishness of the users with the goal of maximizing the overall utility in communication networks. In this scheme the overlay network have modeled as a competitive economy in which the content provided by an origin server can be viewed as commodity and the origin server and the peers who multicast the content to their downside are considered as the firms.

In this paper we inspire from the economic principles to design a high performance active queue management algorithm. The underlying idea of this approach, ECORED, is that the problem of transmission rate control in computer networks can be likened to the supplydemand problem in the economy that supply and demand can be adjusted by pricing strategy. The performance of ECORED 
is further examined under various simulated network environments, and compared to some existing AQM algorithms. Our simulation results show that ECORED achieves shorter response time and hence higher performance.

The rest of this paper is organized as follows. In section 2 , the RED algorithm and its problems are studied. In section 3, economic theory is described briefly and in section 4 ECORED algorithm is offered and its functionality is discussed in details. Section 5 presents implementation and simulations results and compares the performance of ECORED by RED, ARED and NLRED mechanism. Finally, the conclusions are provided in section 6.

\section{RED and its Problems}

RED is the most well-known AQM algorithm that developed by S. Floyd and V. Jacobson in $1993^{2}$, and it is widely implemented in routers today. The RED algorithm is a basis for many other AQM proposals which seek to enhance the performance of the basic RED structure. The primary goal of RED is to achieve low average delay and high throughput. In order to achieve these goals, the RED gateway directs queue by monitoring the average queue size (avg), and when the avg exceeds the expected lower threshold $\min _{t h}$, arriving packets are randomly dropped or marked with a particular probability, in order that some connections can sense the early congestion, and then regulate their own window sizes to avoid serious congestion and packet lost. Once the average queue size is larger than the expected upper threshold max $_{t h}$, the RED mechanism drops or marks every arriving packet. Drop probability is varied linearly from 0 to maxp when the RED queue occupancy is in the region between $\min _{\text {th }}$ and $\max _{\text {th }}$ as shown in Figure 1.

Therefore, the RED algorithm contains two computational parts that are computation of the average queue length avg and calculation of the drop probability $p$.The average queue size of a RED queue is calculated using a

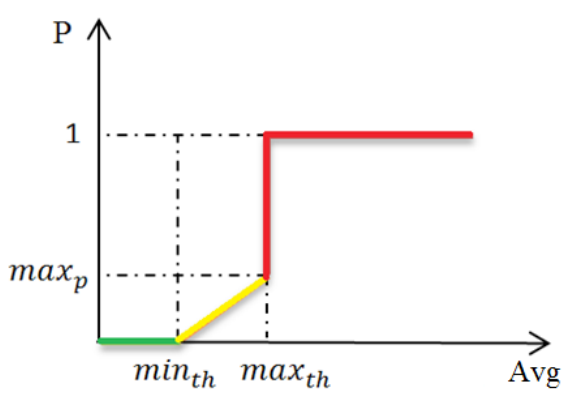

Figure 1. RED Dropping Probability vs. Queue Size². low-pass filter with an exponentially weighted moving average that can be got with the equation (1):

$$
\text { avg }=(1-w) \text { avg }+w q
$$

Where avg is the current average queue size, avg is average queue size at the previous time, $q$ is the current instantaneous queue size and $w$ is exponential averaging weight which is limited at $[0,1]$. avg is updated at the time of packet arrival ${ }^{2}$. Each arriving packet is dropped with probability $p$ given by equation (2):

$$
p=\frac{a v g-\min _{t h}}{\max _{t h}-\min _{t h}} \operatorname{maxp}
$$

Which $\operatorname{maxp}$ is the drop probability when avg is equal to $\max _{t h}, \max _{t h}$ and $\min _{t h}$ are the expected upper and lower thresholds. General algorithm for RED gateways is shown in Figure 2.

RED controls the average queue size within the expected range $\min _{t h}$, $\max _{t h}$, even in the lack of cooperating sources. RED uses only the average queue length as a congestion meter. Because the average queue length considers only long-term behavior of the queue, this algorithm fails to see instantaneous changes of the queue length and hence its reaction to the congestion is not fast enough. In other words the feedback generated by using only the average queue length does not reflect the network congestion precisely and hence leads to low performance. Since, this approach ignores instantaneous changes of the queue length it results in under-utilization of network links and higher packet loss if queues jump between empty and full.

In heavily congested networks RED's congestion notification must be more aggressive, in order to avoid packet loss due to buffer overflow. On the other hand, in lightly congested networks RED's congestion notification must be less aggressive, in order to both preventing excessive packet drop and to maintain link utilization at an

For each packet arrival

1. Calculate the average queue size avg

2. If $\min _{\text {th }} \leq a v g \leq \max _{\text {th }}$

3. Calculate probability $p$

4. Drop/mark the arriving packet with probability $p$

5. Else if $a v g \geq$ max $_{\text {th }}$

6. Drop/Mark the arriving packet

Figure 2. The RED algorithm². 
acceptably high level. But the aggressiveness level of RED significantly depends on setting of its control parameters. But value of these control parameters in original RED algorithm is fixed, and hence as regards network condition varies largely; the invariant aggressiveness of RED is not efficient in dynamic condition of network.

The problem is the difficulty of the parameter setting of RED, that is, we could not find one parameter set to make the RED algorithm work effectively in various network conditions. If RED router is to be effective, its control parameters must be set to values appropriate to the condition of the network (link bandwidth, the number of active connections, congestion level, and so on). As pointed out by other researchers, however, it is very difficult to set them to cope with the dynamically changing network condition.

\section{Some Economic Principles}

Economics is the social science that examines the production, distribution, and consumption of goods and services. As well as, economics is the analysis of how society determines how it will distribute, or allocate, its scarce goods or services to a population and to a set of purposes that has an infinite desire for more. The large set of interrelated economic production and consumption activities which aid in determining how scarce resources are allocated. Scarcity means that society has limited resources and therefore cannot produce all the goods and services people wish to have. Also the problem of resource allocation stems from the scarcity of resources in relation to the demand for them and the consequent need for 'choice'. Allocation of resources is apportionment of productive assets among different uses.

In economics, the term equilibrium is used to suggest a state of "balance" between supply forces and demand forces. On the other word, economic equilibrium is a state in which economic forces are balanced and in the absence of external influences the (equilibrium) values of economic variables will not change. It is the point at which quantity demanded and quantity supplied are equal ${ }^{24}$. For example, an increase in supply will disrupt the equilibrium, leading to lower prices. According to the pricing theory and scarcity principle th e price for a scarce good should rise until equilibrium is reached between supply and demand.

Pricing strategies for products or services encompass three main ways to improve profits. The business owner can cut costs or sell more, or find more profit with a better pricing strategy. When sales are hard to find and costs are already at their lowest, adopting a better pricing strategy is a key option to stay viable. Economic theory asserts that in a free market economy the market price reflects interaction between supply and demand. The price is set so as to equate the quantity being supplied and that being demanded.

In economics, inflation is a rise in the general level prices of goods and services in an economy over a period of time ${ }^{25}$. When the general price level rises, each unit of currency buys fewer goods and services. Consequently, inflation reflects a reduction in the purchasing power per unit of money or a loss of real value in the medium of exchange and unit of account within the economy ${ }^{26}$.

Economic equilibrium as an effective means to achieve economic efficiency for computer networks is considered in this paper. An appropriate pricing policy will lead to equilibrium and therefore improve the overall utilization and performance of the network. A proper pricing policy influences the demands of users and the network revenue.

\section{The ECORED Algorithm}

The TCP/AQM flow dynamics could be modeled by economics theories. One of the most important goals of the congestion control is adjusting the transmission rate with the available bandwidth. We can consider that the scarce good in AQM is the bandwidth and AQM which aid in determining how this scarce resource is allocated. As the underlying idea, ECORED assumes that the problem of transmission rate control in computer networks can be likened to the supply-demand problem in the economy, that supply and demand can be adjusted by a proper pricing strategy.

As shown in Table 1, in our mapping, the network bandwidth can be viewed as the scarce commodity and the behavior of the overlay flows can be mapped to that of

Table 1. Packet loss and average utilization of bottleneck link for various AQM algorithms

\begin{tabular}{ll}
\hline Network & \multicolumn{1}{c}{ Economy } \\
\hline Link Bandwidth & Scarce Commodity \\
Flows & Consumers \\
Congestion & Inflation \\
Drop Probability & Price \\
\hline
\end{tabular}


the consumers in the economy. Also, the congestion can be thought as the inflation of the economy. The drop probability can be considered as the price that it is the feedback generated to reflect the network congestion. Economic equilibrium is the point at which demanded and supplied are equal. In our mapping, the term equilibrium is used to suggest a state of "balance" between transmission rate and available bandwidth, which leads to maximize the aggregated utility of the link in conjunction with minimizing the packet loss. For example, an increase in transmission rate (supply) will disrupt the equilibrium, leading to higher prices. Obviously, the price should depend on the degree of congestion.

As previously mentioned, RED uses only the average queue length as a congestion meter to trigger packet dropping as a congestion feedback. Since the average queue length considers only long-term behavior of any queue, this approach fails to see instantaneous changes of the queue and hence its reaction is not fast enough. This leads to under-utilization of network links and higher packet loss. In ECORED, two meters i.e. queue length growth velocity (QV) and Drop Velocity (DV) are introduced to mitigate such problems. Based on this observation, in addition to average queue length the price is depending on the QV and DV.

$$
\begin{gathered}
\text { demand }=F(\text { pricing }) \\
\text { Pricing }=G(Q V, D V, \text { avg })
\end{gathered}
$$

We believe that a combination of average queue length and QV and DV can make a better feedback that returns more precisely information about the congestion level in the router which is further described in detail herein below.

\subsection{Queue Length Growth Velocity (QV)}

It is clear that in heavy congestion state, the time required for the specified change of queue length is lower than the same time in light congestion state and so the value of queue length growth velocity in heavy congestion is higher than light congestion. If queue length growth velocity is positive that means the queue is growing up and overflow may occur, then the drop probability should be increased, and conversely if it is negative, then the queue may become empty and the drop probability should be decreased. The queue length growth velocity $(\mathrm{QV})$ can be got with the Equation (5):

$$
Q V_{c}=(1-\alpha) Q V_{t-1}+\alpha\left(\frac{q_{t}-q_{t-1}}{T_{t}-T_{t-1}}\right)
$$

Where $q_{t}$ and $T_{t}$ are respectively, the instantaneous queue length in the current interval and the time in the current interval. The constant ' $\alpha$ ' is equal to 0.8 .

Several level of congestion is defined according to the value and sign of the queue length growth velocity, and $\max _{\mathrm{p}}$ is tuned in accordance with these levels. Subsequently the percentage of increase or decrease of the drop probability is dependent on queue length growth velocity. It is clear that this method of tuning the max leads to lower queue oscillation. Detailed operations for ECORED can be found in pseudo code of Figure 3 Where $q_{-} t h$ is the difference of the current instantaneous queue length and previous queue length that is assumed to 3 .

We use a Multiplicative Increase Multiplicative Decrease (MIMD) policy for dynamic tuning of RED's $\max _{\mathrm{p}}$ parameter. $\gamma$ is change according to QV in $[1.2,1.6]$ intervals.

\subsection{Drop Velocity (DV)}

Our algorithm recognizes persistent congestion severity through calculating the velocity of packet drops, and controls the packets drop rate by tuning two thresholds of RED according to packets drop velocity. Total packets that dropped by RED algorithm can be divided into

For each packet arrival

1. $q_{t}=$ current queue length

2. $\Delta \mathrm{q}=q_{t}-q_{t-1}$

3. If $\Delta q>=q_{-} t h$

4. Time $_{t}=$ current time

5. $\Delta t=$ Time $_{t}-$ Time $_{t-1}$

6. Calculate $Q V_{t}$

7. $\mathrm{t}=\mathrm{t}+1$

8. If $\min _{t h} \leq \mathrm{avg} \leq \max _{t h}$

9. If $Q V_{t}>0$

10. //Increment maxp according to the $Q V_{t}$

11. Fix $\operatorname{maxp}=\operatorname{maxp}^{*} \gamma$

12. Else if $Q V_{t}<0$

13. // Decrement max $p$ according to the $Q V_{t}$

14. Fix $\operatorname{maxp}=\operatorname{maxp} / \gamma$

Figure 3. Pseudocode of the ECORED algorithm. 
two categories: first early packet drops which the packets randomly with probability of $\mathrm{p}$ are dropped before buffer overflow occurs; second force packet drops that all arrival packets are dropped after buffer overflow occurs. ECORED differentiates these two, and assumes that existence of force dropped packets is because of low early drop probability.

The value of two thresholds $\min _{\text {th }}$ and $\max _{\text {th }}$ has important role in RED's packet dropping and setting of proper value of these control parameters is main problem of RED algorithm. ECORED uses velocity of packet drop for dynamic tuning of these thresholds. Velocity of packet drops in time $t$ is defined as in Equation 6.

$$
D V_{t}=(1-\beta) D V_{t-1}+\beta\left(\frac{\text { DropCount }_{t}}{\text { Time }_{t}-\text { Time }_{t-1}}\right)
$$

Where DropCount $t_{t}$ shows the number of dropped packets in current interval. Each interval scilicet Time ${ }_{t}-$ Time $_{t-1}$ is $0.5 \mathrm{sec}$. Also in Equation 6, $\beta$ is considered to be 0.2 . Pseudo code of Figure 4 shows how to calculate the QV for each packet arrival.

Several level of persistent congestion is defined according to the value of the DV and existence or nonexistence of force packet drops. Afterwards the value of two thresholds $\min _{\text {th }}$ and $\max _{\text {th }}$ are tuned dynamically in accordance with these levels. Whatever number of defined congestion levels increases, then the accuracy of the calculated packets drop probability will increase, but fluctuation of this probability will increase against. According to the RED's packet dropping, packets drop probability has reverse relation with difference of $\max _{\text {th }}$ and $\min _{\text {th }}$. Thus, with assumption that $\min _{\text {th }}$ is fixed, a

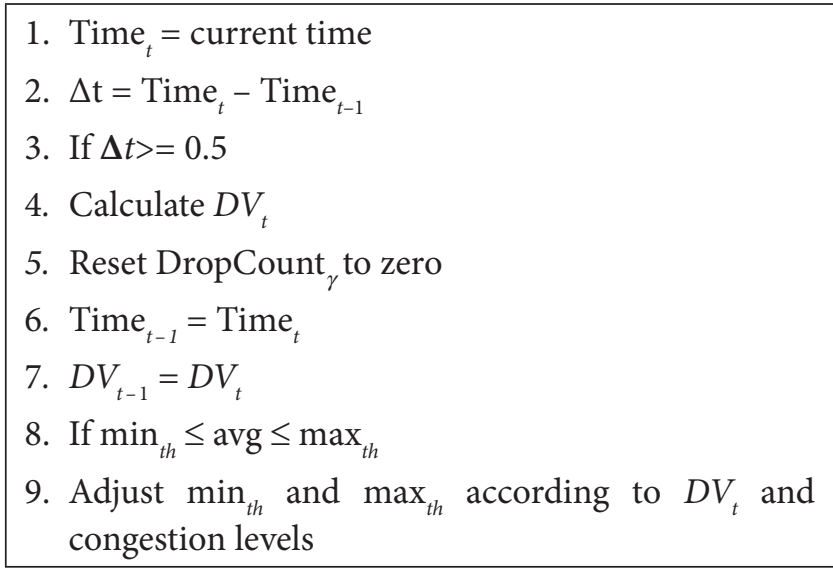

Figure 4. Pseudocode of the ECORED algorithm. decrease of $\max _{\text {th }}$ causes to increase of packets drop probability, and vice versa.

\section{Performance Evaluation of ECORED}

In order to evaluate performance of the proposed mechanism, ECORED implemented by making some modification on RED module of ns-2 software package. In this section we only report one sample results selected from a large set of several experiments and focus on the following key performance metrics: packet loss ratio, utilization and queue length.

Consider a network with a set of 5 source nodes and a set of 5 destination nodes. The network has a simple dumbbell topology as shown in Figure 5. The bottleneck link capacity is $2 \mathrm{Mbps}$ and all sources have the same RTT of $5 \mathrm{~ms}$. The simulations use RED with NS's default values. The simulation experiments were run for 100 seconds.

The results of this comparison show that while these algorithms encountered with the same congestion, ECORED avoids high packet loss and achieves higher utilization. Figure 6 to Figure 8 show the comparison results of these algorithms in terms of utilization, packet loss and queue size.

Figure 6 shows bottleneck utilization for various AQM algorithms. According to this figure, utilization of all algorithms is near optimal value, but ECORED has the best utilization. Since increased drop rate leads to poor performance of the network, any congestion control scheme tries to keep packet loss as low as possible. Packet losses of various algorithms are shown in Figure 7. According to this figure, ECORED drops fewer packets in compare with other algorithms excepting RED and NLRED. Figure 6 shows instantaneous queue length of various algorithms. As we can see in this figure, the queue of RED, ARED and NLRED sometimes become empty and this cause to decrease the bottleneck utilization that ECORED has solved this problem.

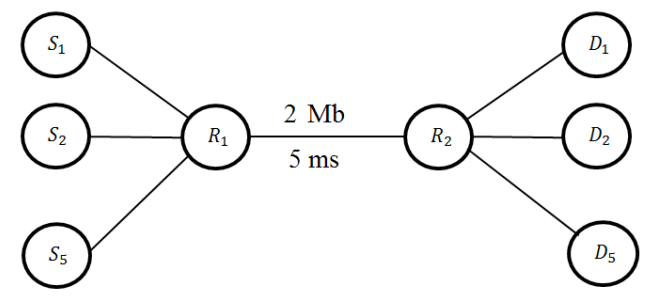

Figure 5. The network topology. 

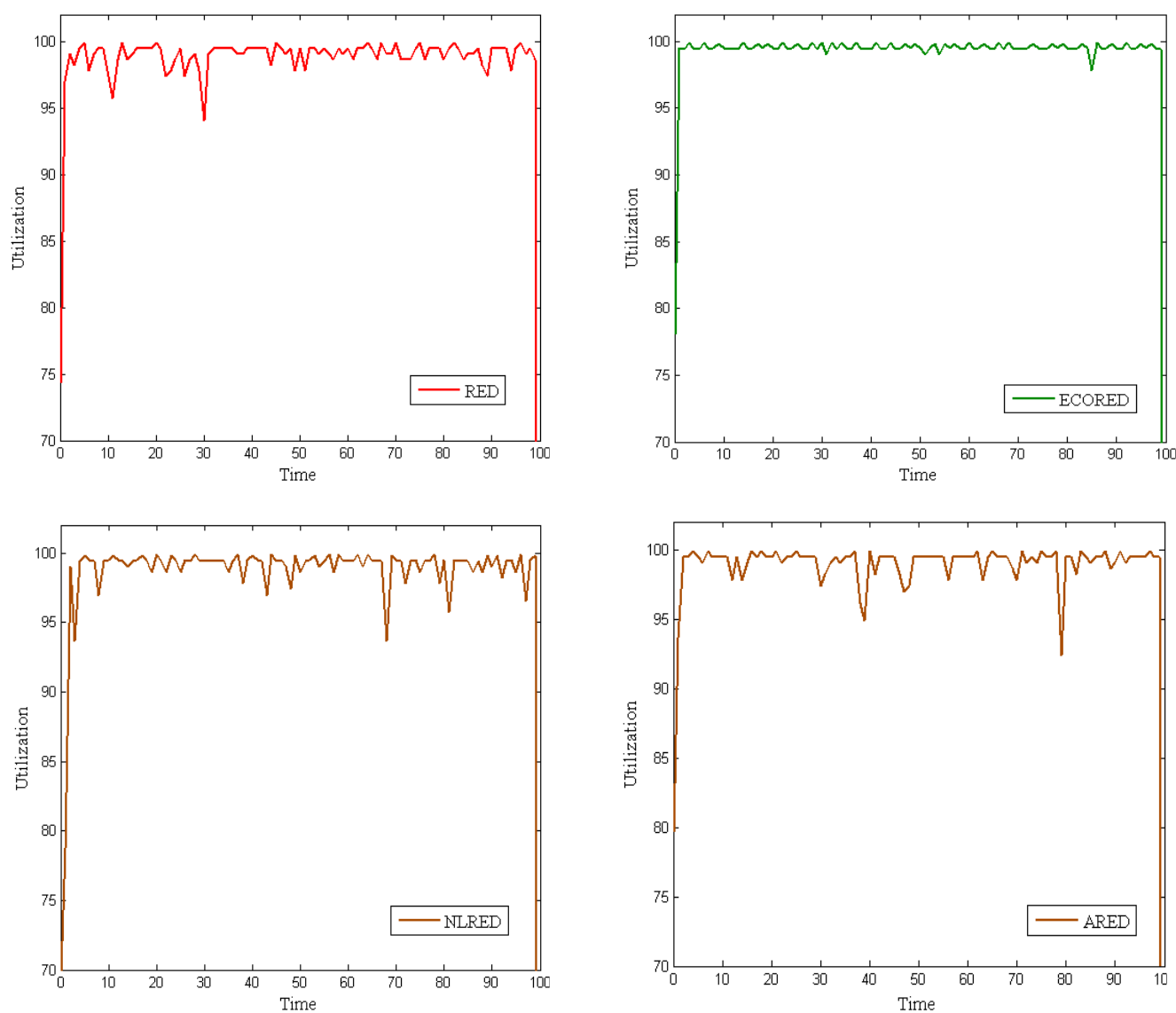

Figure 6. The utilization of ECORED, RED, ARED and NLRED.
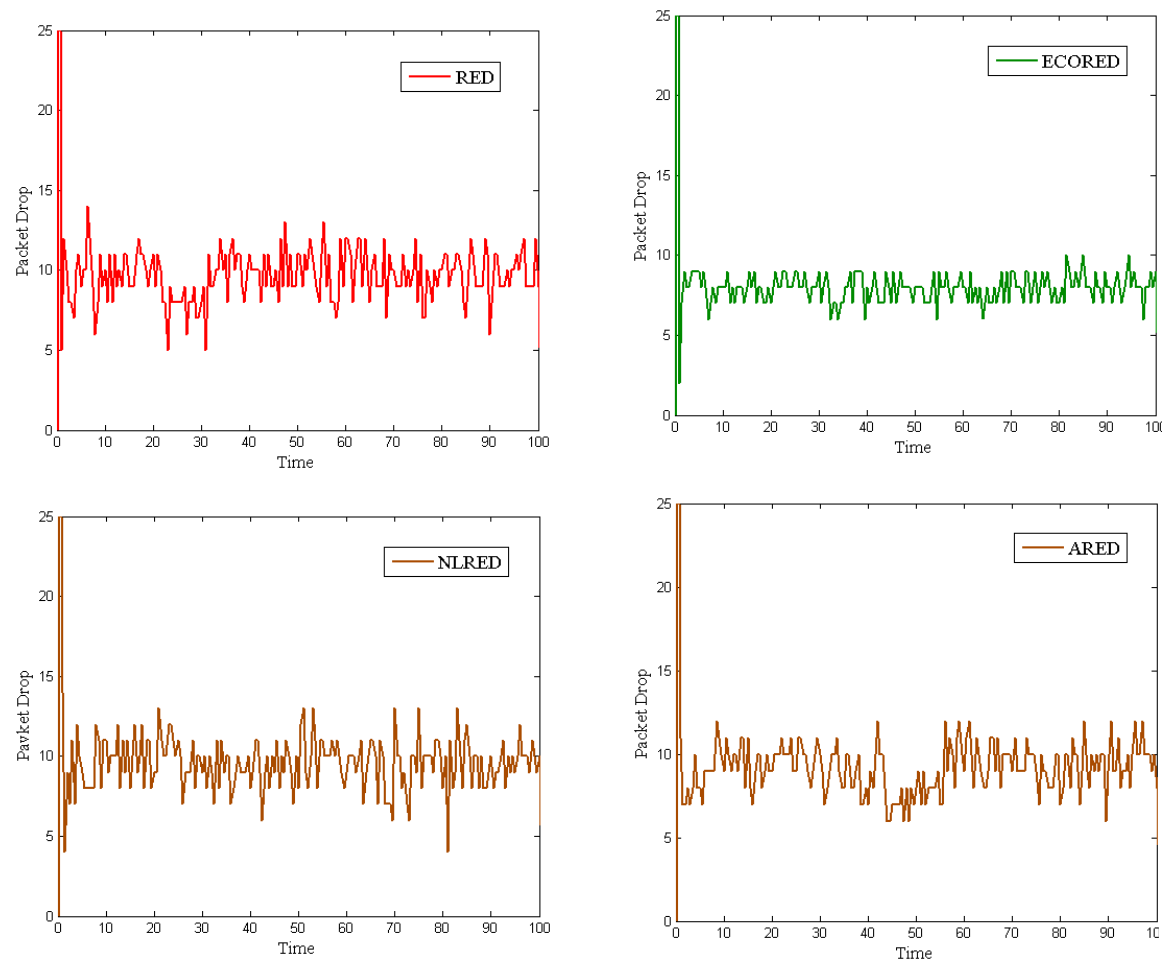

Figure 7. The packet loss of ECORED, RED, ARED and NLRED. 

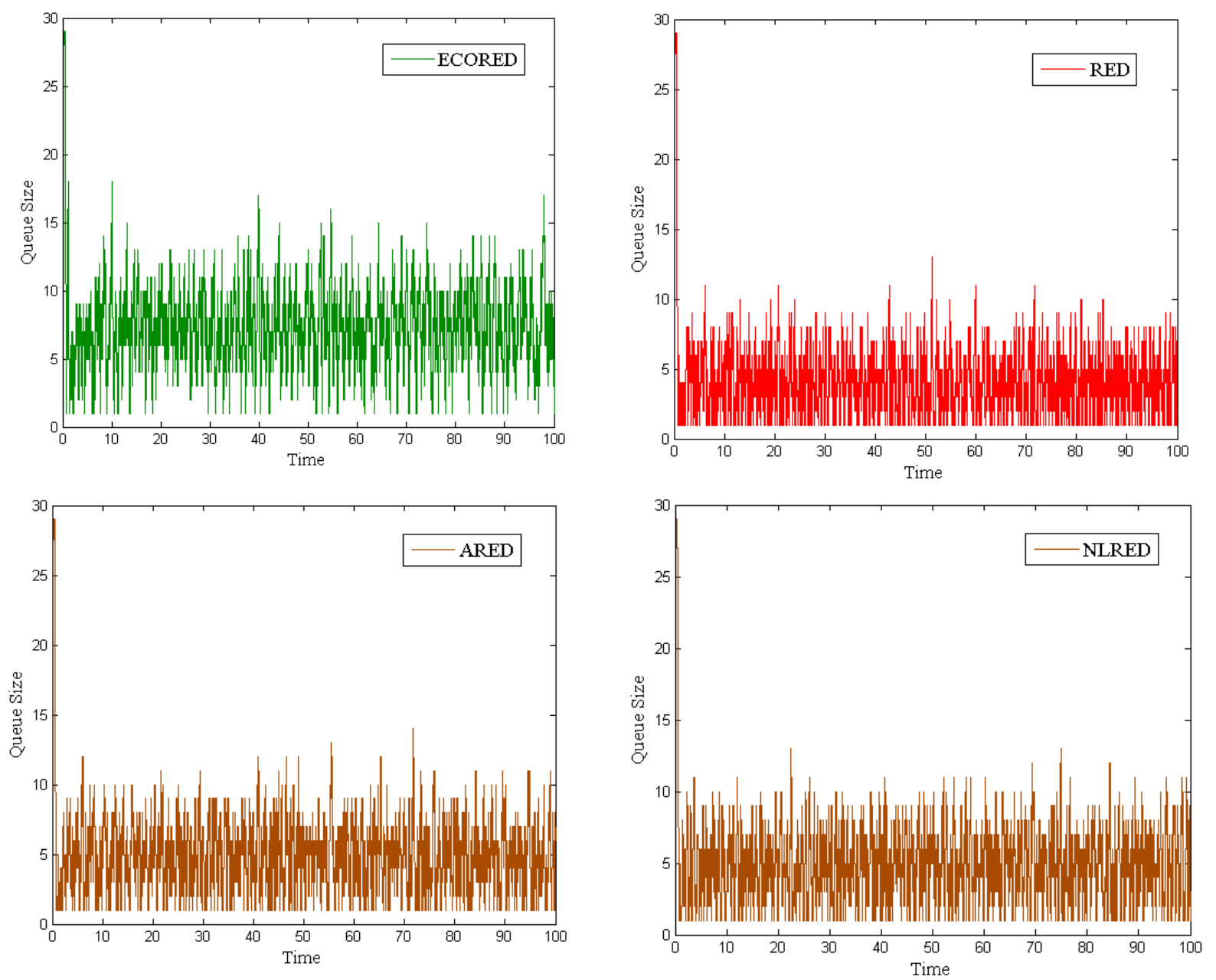

Figure 8. The queue size of ECORED, RED, ARED and NLRED.

Comparison of results shows that ECORED has better dynamic performance than RED, ARED and NLRED. As can be found in these results, ECORED achieves high utilization and also reduces the packet loss considerably. Obviously, detection of congestion in its beginning stages and fast reaction are essential to develop a successful congestion control scheme. ECORED by using both of the queue length growth velocity and the drop velocity in order to detection of congestion in its beginning stages can achieve equilibrium between transmission rate and available bandwidth. This equilibrium is the reason

Table 2. Packet loss and average utilization of bottleneck link for various AQM algorithms

\begin{tabular}{lcccc}
\hline & ECORED & RED & ARED & NLRED \\
\hline Packet loss & 1621 & 1959 & 1867 & 1949 \\
Utilization & 98.39 & 97.76 & 97.91 & 97.64 \\
\hline
\end{tabular}

for low packet loss and high utilization of ECORED as compared to RED, ARED and NLRED.

Table 2 shows the overall results of comparison between ECORED, RED, ARED and NLRED in terms of packet loss and average utilization.

\section{Conclusion}

In this paper, we have proposed an active queue management algorithm by using economic theory, ECORED, which has never been considered in existing AQM algorithms. In ECORED, we have used both of the queue length growth velocity and the drop velocity $\mathrm{n}$ order to achieve equilibrium between transmission rate and available bandwidth. Equilibrium leads to maximize the aggregated utilization of the link along with minimizing the packet loss. We have compared performance of ECORED with some existing AQM algorithms, including 
RED, ARED, and NLRED by using ns-2 simulator. Our results have showed that ECORED outperforms theses algorithm. Furthermore, the ECORED does not change the main principle of the RED algorithm, and can be applied in practice.

\section{References}

1. B. Braden, D. Clark, J. Crowcroft, B. Davie, S. Deering, D. Estrin, S. Floyd, V. Jacobson, G. Minshall, C. Partridge, L. Peterson, K. Ramakrishnan, S. Shenker, J. Wroclawski, and L. Zhang, "Recommendations on Queue Management and Congestion Avoidance in the Internet," Network Working Group RFC 2309. 1998.

2. S. Floyd, V. Jacobson. Random early detection gateways for congestion avoidance. IEEE/ACM Trans. Networking. 1993; 1(4), pp. 397-413.

3. M. May, J. Bolot, C. Diot, and B. Lyles, "Reasons not to deploy RED," In the 7th International Workshop on Quality of Service IWQoS'99 IEEE, 1999 May 31-Jun 4, London, 1999, pp. 260-262.

4. W. Feng, D. Kandlur, D. Saha, and K. G. Shin, "A selfconfiguring RED gateway," in Proc INFOCOM'99 IEEE, 1999 Mar 21-25, New York, 1999, pp. 1320-1328.

5. S. Floyd, R. Gummadi, and S. Shenker, "Adaptive RED: An Algorithm for Increasing the Robustness of RED's Active Queue Management," Preprint, available from http://www. icir. org/floyd/papers.html, 2001.

6. R. J. La, P. Ranjan, and E. H. Abed, "Analysis of adaptive random early dection (Adaptive RED)," in Proc ICT'03, Berlin, Germany, 2003, pp. 279-308.

7. C. Wang, J. Liu, B. Li, K. Sohraby, and Y. T. Hou. LRED: a robust and responsive AQM algorithm using packet loss ratio measurement. IEEE Trans. Parallel and Distributed Systems. 2007; 18(1), pp. 29-43.

8. C.V. Hollot, V. Maisra, D. Towsley, W. Gong, "On designing improved controllers for AQM routers supporting TCP flows," in Proc INFOCOM2001 IEEE, 2001 Apr 22-26, Anchorage, AK, 2001, pp. 1726-1734.

9. L. Tan, W. Zhang, G. Peng, and G. Chen. Stability of TCP/ RED systems in AQM routers. IEEE Trans. Automatic Control. 2006; 51(8), pp. 1393-1398.

10. B. Zheng, M. Atiquzzaman. A framework to determine bounds of maximum loss rate parameter of RED queue for next generation routers. Network and Computer Applications. 2008; 31(4), pp. 429-445.
11. J. Zhang, W. Xu, L. Wang. An improved adaptive queue management algorithm based on nonlinear smoothing. Advanced Materials Research. 2011; 295, pp. 1823-1828.

12. S. Jamali, S. R. Zahedi. An improvement over RED algorithm by using particle swarm optimization. IEICE Electronics Express. 2010; 7(17), pp. 1276-1282.

13. J. Sun, KT. Ko, G. Chen, S. Chan, M. Zukerman. PD-RED: to improve the performance of RED. IEEE Commun. Lett. 2003; 7(8), pp. 406-408.

14. S. Athuraliya, SH. Low, VH. Li, Q. Yin. REM: active queue management. IEEE Network. 2001; 15(3), pp. 48-53.

15. ID. Barrera, S. Bohacek, GR. Arce. Statistical detection of congestion in routers. IEEE Trans. Signal Processing. 2010; 58(3), pp. 957-968.

16. H. Yousefi'zadeh, A. Habibi, X. Li, H. Jafarkhani, C. Bauer. A statistical study of loss-delay tradeoff for RED queues. IEEE Trans. Communications. 2012; 60(7), pp. 1966-1974.

17. K. Chitra, G. Padamavathi. Classification and performance of AQM-based schemes for congestion avoidance. International Journal of Computer Science and Information Security (IJCSIS). 2010; 8(1), pp. 331-340.

18. X. Wang, H. Schulzrinne, "Pricing network resources for adaptive applications in a differentiated services network," in Proc. INFOCOM 2001, Anchorage, AK, 2001, pp. 943-952.

19. M. Baglietto, R. Bolla, F. Davoli, M. Marchese, M. Mongelli. A proposal of new price-based Call Admission Control rules for Guaranteed Performance services multiplexed with Best Effort traffic. Computer Communications. 2001; 26(13), pp.1470-1483.

20. N. Jin, S. Jordan. The effect of bandwidth and buffer pricing on resource allocation and QoS. Computer Networks. 2004; 46(1), pp. 53-71.

21. X. Feng, F. Lau. A new economic generalized particle model for flow control. Computer Networks. 2010; 54(3), pp. 506-524.

22. D. Shuai, X. Feng. The parallel optimization of network bandwidth allocation based on generalized particle model. Computer Networks. 2006; 50(9), pp. 1219-1246.

23. M. Analoui, MH. Rezvani. Microeconomics-based resource allocation in overlay networks by using non-strategic behavior modeling. Communications in Nonlinear Science and Numerical Simulation. 2011;16(1), pp. 493-508.

24. HR. Varian, "Microeconomic analysis," Norton, 3nd ed., vol. 2, New York, 1992.

25. A. Gregson, "Pricing strategies for small business," SelfCounsel Press, 1nd ed., BC, Canada, 2008.

26 K. Monroe, "The Pricing Strategy Audit," Cambridge University Press, Cambridge, United Kingdom, 2000. 